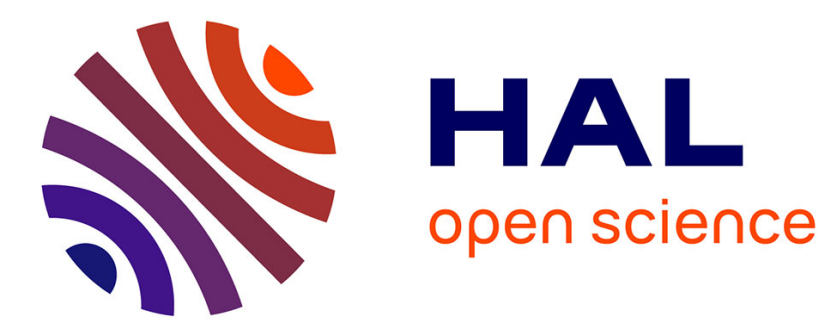

\title{
Glaucoma Detection based on Local Binary Patterns in Fundus Photographs
}

Maya Alsheh Ali, Thomas Hurtut, Timothée Faucon, Farida Cheriet

\section{To cite this version:}

Maya Alsheh Ali, Thomas Hurtut, Timothée Faucon, Farida Cheriet. Glaucoma Detection based on Local Binary Patterns in Fundus Photographs. SPIE Medical Imaging, Feb 2014, United States. pp.903531-903531-7, 10.1117/12.2043098 . hal-00993552

\section{HAL Id: hal-00993552 \\ https://hal.science/hal-00993552}

Submitted on 20 May 2014

HAL is a multi-disciplinary open access archive for the deposit and dissemination of scientific research documents, whether they are published or not. The documents may come from teaching and research institutions in France or abroad, or from public or private research centers.
L'archive ouverte pluridisciplinaire HAL, est destinée au dépôt et à la diffusion de documents scientifiques de niveau recherche, publiés ou non, émanant des établissements d'enseignement et de recherche français ou étrangers, des laboratoires publics ou privés. 


\title{
Glaucoma Detection based on Local Binary Patterns in Fundus Photographs
}

\author{
Maya Alsheh Ali ${ }^{a, b}$, Thomas Hurtut ${ }^{a, b}$, Timothée Faucon ${ }^{c}$, Farida Cheriet ${ }^{b}$ \\ ${ }^{a}$ LIPADE, University of Paris Descartes, France; \\ ${ }^{b}$ École Polytechnique de Montréal, Canada; \\ ${ }^{c}$ DIAGNOS inc., Montréal, Canada
}

\begin{abstract}
Glaucoma, a group of diseases that lead to optic neuropathy, is one of the most common reasons for blindness worldwide. Glaucoma rarely causes symptoms until the later stages of the disease. Early detection of glaucoma is very important to prevent visual loss since optic nerve damages cannot be reversed. To detect glaucoma, purely data-driven techniques have advantages, especially when the disease characteristics are complex and when precise image-based measurements are difficult to obtain. In this paper, we present our preliminary study for glaucoma detection using an automatic method based on local texture features extracted from fundus photographs. It implements the completed modeling of Local Binary Patterns to capture representative texture features from the whole image. A local region is represented by three operators: its central pixel (LBPC) and its local differences as two complementary components, the sign (which is the classical LBP) and the magnitude (LBPM). An image texture is finally described by both the distribution of LBP and the joint-distribution of LBPM and LBPC. Our images are then classified using a nearest-neighbor method with a leave-one-out validation strategy. On a sample set of 41 fundus images (13 glaucomatous, 28 non-glaucomatous), our method achieves $95.1 \%$ success rate with a specificity of $92.3 \%$ and a sensitivity of $96.4 \%$. This study proposes a reproducible glaucoma detection process that could be used in a low-priced medical screening, thus avoiding the inter-experts variability issue.
\end{abstract}

Keywords: Glaucoma, texture features, computer aided diagnosis, Local Binary Patterns, Fundus photographs.

\section{INTRODUCTION}

Glaucoma, a group of diseases that lead to optic neuropathy, is one of the most common reasons for blindness worldwide. Glaucoma rarely causes symptoms until the later stages of the disease, and epidemiology surveys in North America and Europe have shown that approximately $50 \%$ of cases are undetected ${ }^{1}$ Early detection and prevention is the only way to treat glaucoma and avoid total loss of vision since the optic nerve damages from glaucoma cannot be reversed.

Doctors diagnose glaucoma based on several factors: the patient's family history, intraocular pressure, central corneal thickness, appearance of the anterior chamber angle, optic nerve appearance, optic nerve structure including the nerve fiber layer, and optic nerve function. Currently, a diagnosis can't be made based on a single finding. For example, high intraocular pressure is a primary thing to look for to detect glaucoma, and yet a third of glaucoma patients have normal eye pressure ${ }^{[2}$ Most glaucoma tests are very time consuming and also need glaucoma experts and diagnosis equipment. Therefore, new automatic techniques to diagnose glaucoma at early stages are needed with both accuracy and speed.

There are several obstacles to automate glaucoma diagnosis. ${ }^{[2}$ First of all, there is no expert consensus for early glaucoma symptoms and the gold standard in diagnosis still relies on expert decision based on fundus images. Another issue is the non-uniformity of the optic disk anatomy combined with the highly variable damages induced by the disease on optic nerves that increase the diagnosis complexity. Glaucomatous and normal eyes have a very close appearance making a definitive diagnosis very difficult to pronounce.

Imaging systems, such as color fundus camera, optical coherence tomography (OCT), Heidelberg retina tomography (HRT) and scanning laser polarimetry, have been extensively used for eye diagnosis. HRT, and OCT can show retina nerve fiber damage even before any loss of vision. However, those equipment are very expensive. Digital fundus photographs show information similar to what ophthalmologists see using their ophthalmoscope; the Heidelberg Retina Tomograph

Further author information: (Send correspondence to Maya Alsheh Ali)

E-mail: maya.alsheh.ali@gmail.com, Telephone: (+33) 183945824 
produces reflectance and topographic images of the retinal surface using confocal laser scanning; Optical Coherence Tomography captures 3D information about the different cell/tissue layers of the retina. According to a study ${ }^{3}$ these devices perform no better than fundus ONHPs (Optic Nerve Head Photographs) to distinguish normal eyes from those with early to moderate glaucoma. Hence fundus cameras can be considered as an appropriate device to be used since it is a low priced imaging device that is widely available and commonly applied for basic eye examinations.

Various techniques in glaucoma detection using fundus photographs have been proposed in the literature ${ }^{4-10}$ Some of them are based on image segmentation of the optic disk structure, usually for cup/disc ratio (CDR) measurement ${ }^{4 / 10}$ The drawback of segmentation-based techniques is that small errors in localization may lead to significant errors in the measurements and consequently in the diagnosis. Besides, a measurement of CDR alone is insufficient and may be misleading as small discs will have smaller cups and hence a smaller CDR ${ }^{[11}$ Other methods use texture analysis to detect retinal nerve fiber layer defects, targeting a single symptom of the disease $\frac{5.77 .9}{15}$ In addition, an appearance-based pattern-recognition approach was proposed to generate glaucoma risk index $!^{6}$ All the existing methods use several complex preprocessing steps to remove the variations which are not related to the disease like illumination correction or histogram equalization. Also blood vessels are removed although they are affected by glaucoma: the location of the vessels changes due to the dying and vanishing of tissue structure. Thus they should be taken into consideration for diagnosis.

Purely data-driven techniques have advantages, especially when the disease characteristics are complex and when precise image-based measurements are difficult to obtain. ${ }^{[}$In this paper, we present a preliminary study of glaucoma detection based on texture analysis using Local Binary Patterns (LBP). It uses the information from the whole image without targeting any specific symptoms. Every local region is represented by three operators: its central pixel (LBPC) and its local differences as two complementary components, the sign (which is tha classical LBP) and the magnitude (LBPM). The discriminative textural information is then captured by both the distribution of LBP and the joint-distribution of LBPM and LBPC.

\section{METHOD}

Red-free fundus images improve the contrast of retina structures, such as blood vessels and retinal nerve fiber layer. Hence, in our study, we use the mean of green and blue channels, Figure 1 . The texture information is captured using features based on Local Binary Patterns. Figure. 2 shows the framework of the proposed method. In the following sections, we present, in one hand a brief review of LBP and its complete modeling followed by parameters discussion, and in the other hand the method used for classification.

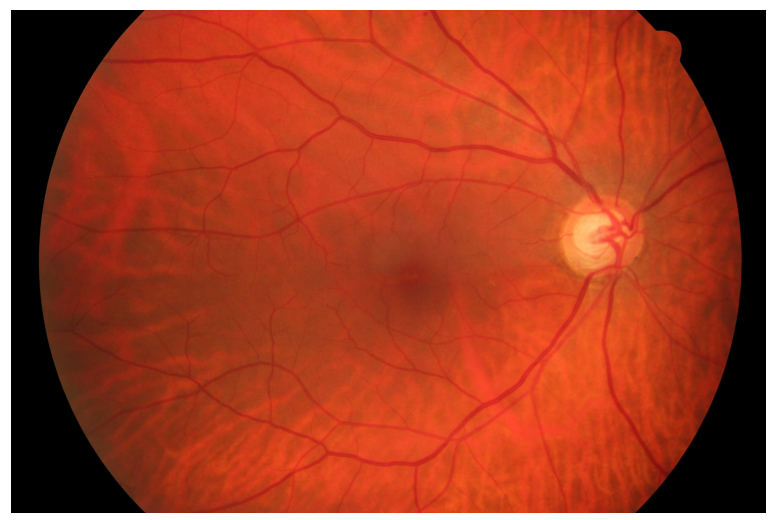

colored glaucomatous image

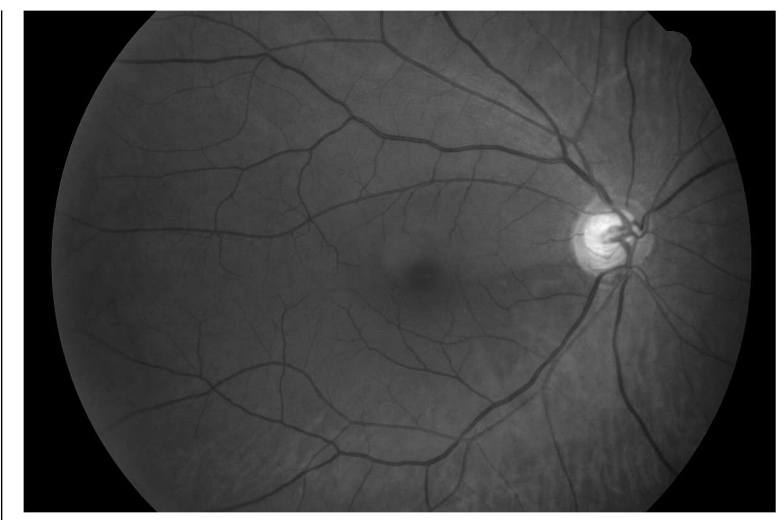

mean of green and blue channels

Figure 1. Example of a colored glaucomatous image and its mean of green and blue channels. This figure shows the improvement in the retina structures contrast using the red free image.

\subsection{Local Binary Pattern}

The local binary pattern operator, proposed by Ojala et al.,12 is a simple and powerful gray-scale invariant method of analyzing textures $\sqrt{13 / 14}$ This operator combines characteristics of statistical and structural texture analysis: it describes the texture with micro-primitives and their statistical placement rules. 
Given a central pixel in the image, a pattern number is computed by comparing its value with those of its circular neighborhood:

$$
L B P_{P, R}=\sum_{p=0}^{P-1} s\left(g_{p}-g_{c}\right) \times 2^{p}, \quad s(x)= \begin{cases}1 & \text { if } x \geq 0 \\ 0 & \text { otherwise }\end{cases}
$$

where $g_{c}$ is the central pixel gray level value and $g_{p}$ is the one of the $p^{\text {th }}$ neighbor, $P$ is the number of neighbors and $R$ the neighborhood radius. When the coordinates of $g_{c}$ are $(0,0)$, the coordinates of $g_{p}$ are given by $(R \cos (2 \pi p / P, R \sin (2 \pi p / P))$. Suppose the texture image is of size $N \times M$ pixels. After identifying the LBP pattern of each pixel $(i, j)$, the whole texture image is represented by a histogram of LBPs:

$$
\begin{array}{r}
H(k)=\sum_{i=1}^{N} \sum_{j=1}^{M} f\left(L B P_{P, R}(i, j), k\right), \quad k \in[1, K] \\
f(x, y)= \begin{cases}1 & \text { if } x=y \\
0 & \text { otherwise }\end{cases}
\end{array}
$$

where $K$ is the maximal LBP pattern value. The number of possible local patterns $K$ increases rapidly when the number of the considered neighbors $P$ grows. For example with 16 neighbors, the size of the histogram would be $2^{16}$ bins, which is impractical. To get over this problem, Mäenpää et al ${ }^{\sqrt{14}}$ had shown that texture information is mostly contained in patterns which have at most two binary transitions in the circular representation, called uniform patterns. These uniform LBPs reduce the feature dimension from $2^{P}$ to $P(P-1)+3$ and retains most of the image information.

$$
U\left(L B P_{P, R}\right)=\left|s\left(g_{P-1}-g_{c}\right)-s\left(g_{0}-g_{c}\right)\right|+\sum_{P=1}^{P-1}\left|s\left(g_{P}-g_{c}\right)-s\left(g_{P-1}-g_{c}\right)\right|
$$

\subsection{Completed Modeling of LBP}

A completed modeling of LBP was proposed by Guo et al ${ }^{[15}$ to expand the conventional LBP with both the magnitude component (LBPM), defined in Equation 4 and the central pixel intensity (LBPC), see Equation 5 :

$$
\begin{gathered}
L B P M_{P, R}=\sum_{p=0}^{P-1} t\left(g_{p}-g_{c}, T\right) \times 2^{p}, \quad t(x, T)= \begin{cases}1 & \text { if } x \geq T \\
0 & \text { otherwise }\end{cases} \\
L B P C_{P, R}=t\left(g_{c}, T_{I}\right)
\end{gathered}
$$

where $T$ is a threshold set to the mean value of $\left(g_{p}-g_{c}\right)$ from the whole image and $T_{I}$ is the average gray level of the whole image. The three operators are encoded using the same binarization strategy to allow them to be merged.

The image features we use are the distribution of the LBP and the joint distribution of both LBPM and LBPC. We observed that this combination is the most discriminant among all the possible combinations of these three operators.

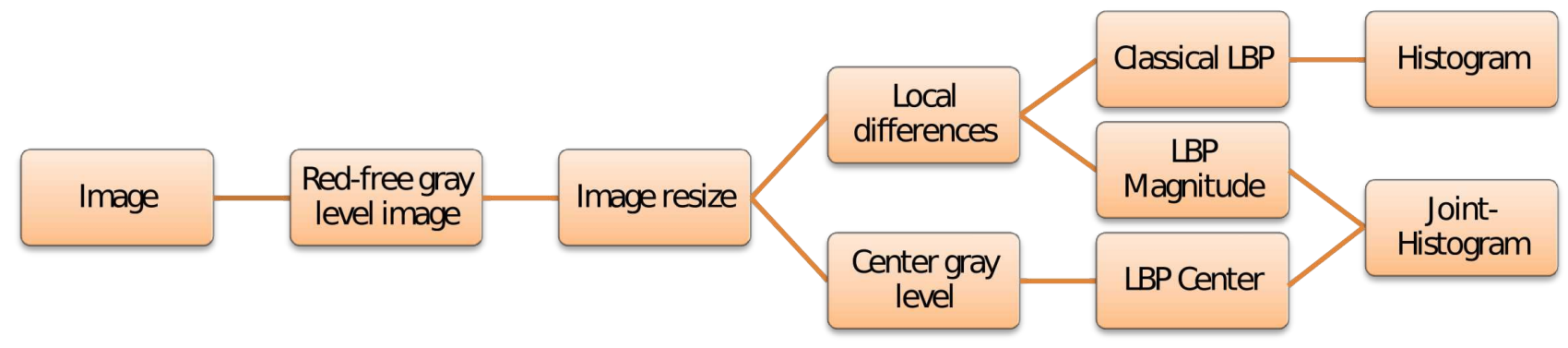

Figure 2. Framework of the proposed method 


\subsection{LBP Parameters}

In order to measure the texture at the most discriminative scale for glaucoma detection, three dependent parameters have to be properly set:

The number of neighbors $P$ : The LBP operator use $P$ neighbors and compare their gray levels with the one of the central pixel, reflecting the intensity gradient on different directions. Besides, the number of neighbors decides the histogram dimension.

The neighborhood radius $R$ : This radius decides the distance between the central pixel and the neighbors to be considered. We have to choose the distance that accurately describe the gray level changes caused by the glaucoma.

The image resolution: The resolution affects the image gradient, since the same image at different resolutions gives different gradient magnitudes. Thus, the resolution radically decides the gradient of the whole image along with the scale of the measured information.

In this study, we empirically choose the parameter values and the best results are obtained with $P=8, R=1$ and with image resolution reduced to $225 \times 225$.

\subsection{Classification}

In order to mitigate the limited size of our database, we use the leave-one-out validation method with a k-nearest neighbor classification. The k-nearest neighbor algorithm is amongst the simplest of all machine learning algorithms: an object is classified by a majority vote of its neighbors, with the object being assigned to the class most common amongst its $\mathrm{k}$ nearest neighbors. If $\mathrm{k}=1$, then the object is simply assigned to the class of that single nearest neighbor.

The inter-feature distance is measured with the Hellinger f-divergence metric. For two discrete probability distributions $P=\left(p_{1} \ldots p_{k}\right)$ and $Q=\left(q_{1} \ldots q_{k}\right)$, their Hellinger distance is defined as:

$$
H(P, Q)=\frac{1}{\sqrt{2}} \sqrt{\sum_{i=1}^{k}\left(\sqrt{p_{i}}-\sqrt{q_{i}}\right)^{2}}
$$

\section{RESULTS}

The images we used for validation are collected from both a public database and a private set from the DIAGNOS company* The public database ${ }^{\dagger}$ contains 30 images of non glaucomatous patients (15 healthy and 15 with diabetic retinopathy), and 15 images of glaucomatous patients of high resolution. DIAGNOS database contains 9 glaucomatous images and 22 images of healthy patients taken by different cameras with both resolution and illumination variability. To avoid the inclusion of both eyes from the same patient, only images of left eyes were employed in this study, although our method can be applied to images of right eyes without any modifications. The final database includes 13 glaucoma images and 28 non glaucomatous images. Figure. 3 shows samples of this database.

Tabel 1 shows our experiments results on our database using the combination of the classical LBP histogram with the joint histogram of magnitude and center. Our method achieves $95.1 \%$ success rate with a specificity of $92.3 \%$ and a sensitivity of $96.4 \%$. It points out the efficiency of the completed model of the LBP for the diagnosis of glaucoma. It is also interesting to note that all the diabetic retinopathy images are correctly classified as non-glaucomatous, emphasizing the descriptor robustness to the widespread diabetic retinopathy.

We present the misclassified images in Figure. 4 with their nearest neighbor images. We can see the high similarity of these images. It is also interesting to note that it is the same healthy image who cause the misclassification.

${ }^{*}$ Note that the most used databases in the literature such as Origa ${ }^{- \text {light }}$ are no more publicly available.
${ }^{\dagger}$ Can be found online: http://www5.cs. fau.de/research/data/fundus-images/ 


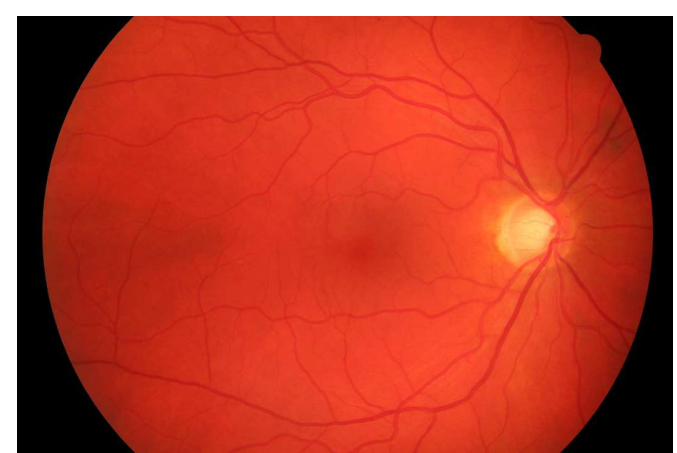

Healthy

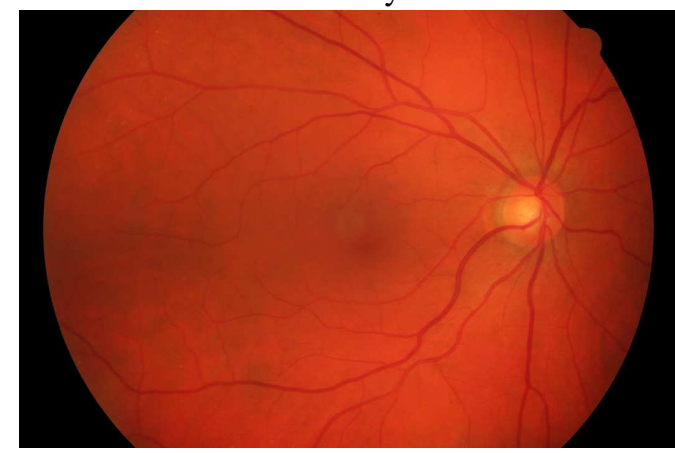

Glaucomatous

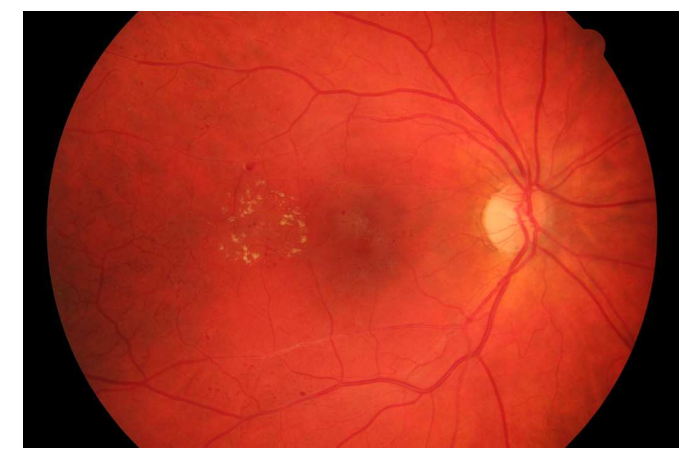

Diabetic Retinopathy

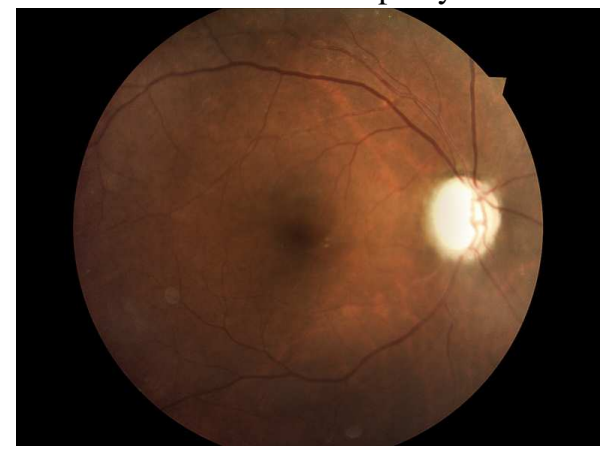

Glaucomatous

Figure 3. Samples from our database: non-Glaucomatous images in the first row and Glaucomatous images in the second row. This figure shows the strong visual overlap between healthy and glaucoma fundus and the distortions induced by the diabetic retinopathy.

Table 1. Statistical results.

\begin{tabular}{|c|c|c|c|c|c|}
\hline Non-glaucomatous & 27 & $\begin{array}{c}1 \\
12\end{array}$ & Specificity & Sensitivity & Recognition Rate \\
\hline & Non-glaucomatous & $\begin{array}{c}12 \\
\text { Glaucomatous }\end{array}$ & $92.3 \%$ & $96.4 \%$ & $95.1 \%$ \\
\hline
\end{tabular}

\section{DISCUSSION AND CONCLUSION}

In this paper, we present an automatic method for glaucoma detection based on textural features. In contrast to the previous work, this method does not require manual assistance, complex preprocessing (such as illumination correction), morphological operations nor segmentation. It implements the completed modeling of Local Binary Patterns to capture representative texture features from the whole red-free image. These features have many attractive properties: Invariant to monotonic gray-scale variations, low complexity, few parameters to be fixed before the feature extraction and satisfactory discriminating power. Robustness in the varying imaging and lighting conditions in addition to the very fast feature extraction are also important in this application. It is also not based on the detection of any specific disease symptom. In addition, this method is robust to the presence of diabetic retinopathy.

In the present paper, we set the relevant LBP parameters empirically. However, such tests might be constrained by the database and thus lack of generalization ability. Future work will include an automatic system to determine the optimal value of these parameters, as well as perform an extensive validation to assess its reliability in clinical screening.

\section{Acnkowledgments}

This work has been partially supported by the ANR SPIRIT \#11-JCJC-008-01 and a NSERC Collaborative Research and Development grant. 


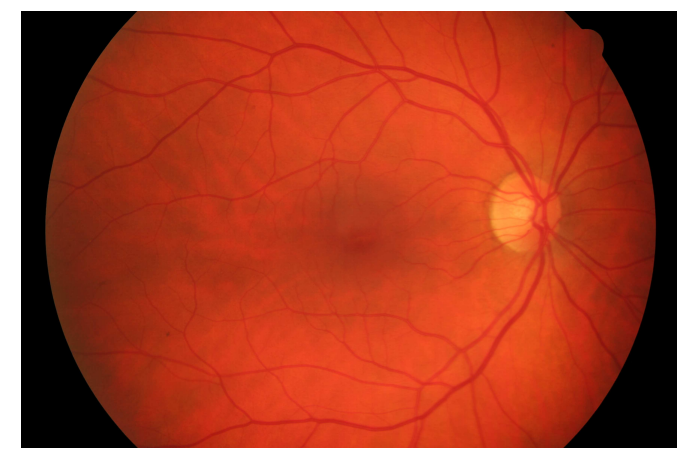

(a) healthy

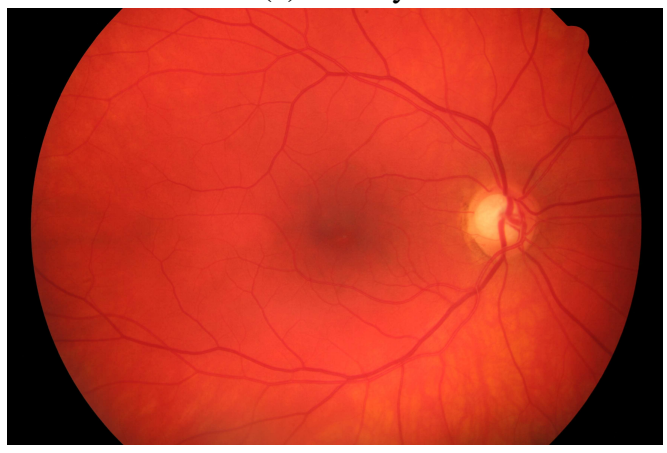

(c) glaucomatous

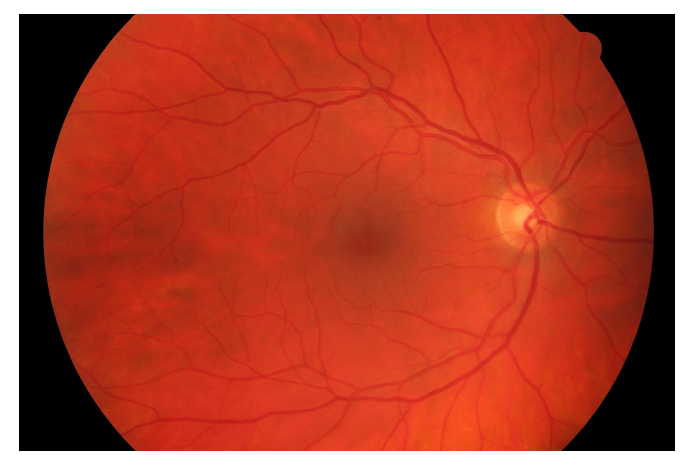

(b) glaucomatous

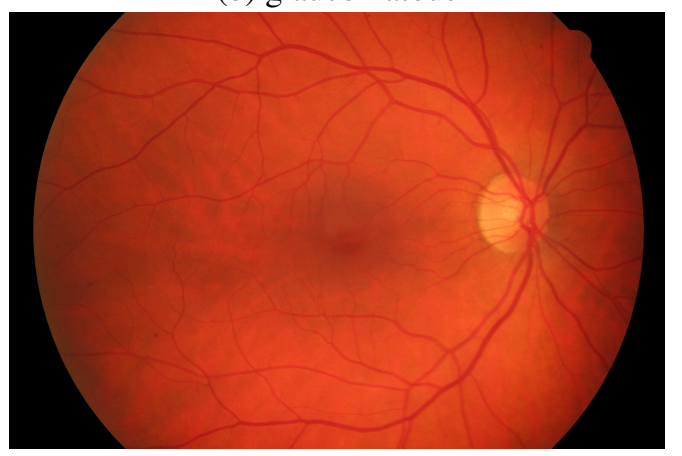

(d)healthy

Figure 4. shows the misclassified images (a) and (c), and their nearest neighbours (b) and (d). Note that the healthy images (a) and (d) are the same.

\section{REFERENCES}

[1] Yu, J., Abidi, S. S. R., Artes, P., McIntyre, A., and Heywood, M., "Diagnostic support for glaucoma using retinal images: a hybrid image analysis and data mining approach," Studies in health technology and informatics 116, 187 (2005).

[2] Kent, C., "Automated glaucoma diagnosis: Any time soon?," Review of Ophtolmology 15(6), 35 (2008).

[3] Greaney, M. J., Hoffman, D. C., Garway-Heath, D. F., Nakla, M., Coleman, A. L., and Caprioli, J., "Comparison of optic nerve imaging methods to distinguish normal eyes from those with glaucoma," Investigative Ophthalmology \& Visual Science 43(1), 140-145 (2002).

[4] Joshi, G., Sivaswamy, J., and Krishnadas, S. R., "Optic disk and cup segmentation from monocular color retinal images for glaucoma assessment," IEEE Transactions on Medical Imaging 30(6), 1192-1205 (2011).

[5] Hayashi, Y., Nakagawa, T., Hatanaka, Y., Aoyama, A., Kakogawa, M., Hara, T., Fujita, H., and Yamamoto, T., "Detection of retinal nerve fiber layer defects in retinal fundus images using gabor filtering," SPIE Medical Imaging 65142, 65142Z-65142Z (2007).

[6] Bock, R., Meier, J., Nyúl, L. G., Hornegger, J., and Michelson, G., "Glaucoma risk index:automated glaucoma detection from color fundus images," Medical Image Analysis 14(3), 471 - 481 (2010).

[7] Acharya, U., Dua, S., Du, X., Vinitha Sree, S., and Chua, C. K., "Automated diagnosis of glaucoma using texture and higher order spectra features," Information Technology in Biomedicine, IEEE Transactions on 15(3), 449-455 (2011).

[8] Dua, S., Acharya, U., Chowriappa, P., and Sree, S., "Wavelet-based energy features for glaucomatous image classification," Information Technology in Biomedicine, IEEE Transactions on 16(1), 80-87 (2012).

[9] Odstrcilik, J., Kolar, R., Jan, J., Gazarek, J., Kuna, Z., and Vodakova, M., "Analysis of retinal nerve fiber layer via markov random fields in color fundus images," 19th International Conference on Systems, Signals and Image Processing (IWSSIP) , 504-507, IEEE (2012).

[10] Nayak, J., Acharya, R., Bhat, P. S., Shetty, N., and Lim, T.-C., "Automated diagnosis of glaucoma using digital fundus images," Journal of medical systems 33(5), 337-346 (2009). 
[11] Bourne, R. R., “The optic nerve head in glaucoma," Community Eye Health Journal 19(59), 44-45 (2006).

[12] Ojala, T., Pietikäinen, M., and Harwood, D., "A comparative study of texture measures with classification based on featured distributions," Pattern recognition 29(1), 51-59 (1996).

[13] Ojala, T., Pietikainen, M., and Maenpaa, T., "Multiresolution gray-scale and rotation invariant texture classification with local binary patterns," Pattern Analysis and Machine Intelligence, IEEE Transactions on 24(7), 971-987 (2002).

[14] Mäenpää, T., Ojala, T., Pietikäinen, M., Soriano, M., Mäenpää, T., Ojala, T., Pietikäinen, M., and Soriano, M., "Robust texture classification by subsets of local binary patterns," Proceedings of the 15th International Conference on Pattern Recognition 3, 935-938 (2000).

[15] Guo, Z., Zhang, D., and Zhang, D., "A completed modeling of local binary pattern operator for texture classification," Image Processing, IEEE Transactions on 19(6), 1657-1663 (2010). 